\title{
Deciphering Long Covid: Next Emergent Healthcare Dilemma
}

\author{
Sana Abbas, Beenish Abbas, Haifsa Rafique, Aisha Rafique, Saima Zafar, and Ramesha Azam
}

\section{ABSTRACT}

Objective: To determine the prevalence of Long Haul COVID-19 Syndrome among Pakistani Population.

Methods: This cross-sectional analytical study was conducted after approval from the ethical review committee of the Foundation University College of Dentistry, Islamabad from June 2021 to September 2021. A nonprobability consecutive sampling methodology was employed and a total of (168) participants after voluntary consent were enrolled in the study, after explaining the study protocol to the patients. The final Questionnaire is comprised of three sections. Section - I focused on the demographic profile (Age, Gender, Profession) of the participants. Section - II was designed to investigate COVID - 19 disease history, comorbid, coronavirus disease severity along with the number of individuals infected in the family, duration of positive polymerase chain reaction results, and management destination (Hospital or Home). Section - III addressed the frequency of persistent symptoms (Gastrointestinal, Systemic, Cardiovascular, Musculoskeletal, Immunological, Dermatological, Neuropsychiatric, Ophthalmological, Pulmonary, Otorhinolaryngological, Endocrine, Reproductive and Genitourinary systems).

Results: 168 responders participated in this survey study, $48(28.6 \%)$ males while 120 (71.4\%) females. About 36 (11.9\%) were found to be suffering from comorbidity, $84(50.0 \%)$ suffered from mild disease symptoms, while only $4(2.4 \%)$ experienced the severe disease. The disease was managed at home for a major chunk of responders, $140(83.3 \%) .132(78.6 \%)$ were found to have suffered from long COVID syndrome with the persistence of symptoms beyond four weeks of infection. The most common symptoms included fatigue $(\mathbf{7 8 . 8} \%)$, fever $(\mathbf{6 0 . 0} \%)$, loss of taste $(57.8 \%)$, dry cough $(45.5 \%)$, headache $(42.4 \%)$, post-exertional malaise $(45.5 \%)$, shortness of breath $(39.4 \%)$, insomnia $(39.0 \%)$, loss of smell $(36.4 \%)$, loss of appetite $(33.3 \%)$ and depression (33.3\%).Male gender $(p=0.009)$, with existing chronic illnesses $(p<0.001)$, moderate and severe disease $(p<0.001)$, higher age group $(\mathrm{p}<0.001)$ and isolated at hospital $(\mathrm{p}=\mathbf{0 . 0 0 2})$.

Conclusion: In conclusion, this study has shown that persistence of COVID disease symptoms (most common symptoms included fatigue, fever, loss of taste, dry cough, headache, post-exertional malaise, shortness of breath, insomnia, loss of smell, loss of appetite, and depression) are common in Pakistani population. Male gender, existing chronic illnesses, moderate and severe disease, higher age group, and isolation at the hospital are the probable risk factors.

Keywords: COVID-19, long, symptoms.
Submitted : October 6, 2021

Published : November 16, 2021

ISSN: $2593-8339$

DOI: $10.24018 /$ ejmed.2021.3.6.1096

\section{S. Abbas*}

National University of Medical Sciences, Pakistan.

(e-mail:

doctor_amcollian@yahoo.com)

B. Abbas

Foundation University Islamabad, Pakistan.

(e-mail:

beenishabbas1982@gmail.com)

H. Rafique

Fauzia Ruth Pfau Hospital, Pakistan. (e-mail: haifsa.rafique@gmail.com)

A. Rafique

National University of Medical

Sciences, Pakistan.

(e-mail: mubasshir85@live.com)

S. Zafar

Foundation University College of Dentistry, Pakistan.

(e-mail: saima_i_khan@hotmail.com)

R. Azam

Foundation University College of

Dentistry, Pakistan.

(e-mail: ramesha.12@hotmail.com)

\section{INTRODUCTION}

Long COVID is defined as the persistence of symptoms for longer than usual (minimum four weeks) in individuals who recovered from COVID-19 [1], [2]. This unusual symptomatology is reported with anecdotal experiences through health care workers, patient groups, and case studies. Hence the Royal College of General Practitioners emphasized on streamlined approach due to the expectant rise in the influx of long haulers [3]. Evidence indicates the prevalence of significant health problems like extreme tiredness, anxiety, mental health problems, reduced muscle function, and post-traumatic stress disorder with subsequent impact on quality of life therefore personalized support is recommended for these patients [4].

More than a year and a half have passed but pathogenesis, symptomatology, duration, and management of long COVID-19 syndrome is still a mystery. Carvalho-Schneider et al.in their recent study reported severity of disease and age (40-59 years) represent greater propensity [5]. Whereas 
comorbidities, body mass index, ethnicity, smoking status, gender, and level of physical activity need to be evaluated for correlation [6], [7].

Direct viral invasion, hyperactivation of the immune response, thrombosis, and hormone imbalances, and inflammatory response causing multiorgan dysfunction are proposed mechanisms reported by [8] Myasthenia gravis, Guillain-Barre syndrome, and postural orthostatic tachycardia triggered by COVID-19 infection and result in the long haul [9], [10].

The rationale of this study was to evaluate the prevalence of long COVID syndrome in the Pakistani population so that efforts in hand could be established to tackle this aspect in addition to issues of acute disease and mass vaccination campaigns.

\section{METHODS}

\section{A. Study Design}

The cross-sectional analytical study was conducted after approval from the ethical review committee of the Foundation University College of Dentistry, Islamabad from June 2021 to September 2021.

\section{B. Sampling Methods}

The sample size (98) was calculated by keeping a level of significance $95 \%$ confidence interval, $5 \%$ margin of error, and anticipated $(93.2 \%)$ prevalence of persistent symptoms characteristic of long COVID syndrome as elaborated by a study conducted by [11].

A non-probability consecutive sampling methodology was employed and a total of (168) participants after voluntary consent were enrolled in the study, after explaining the study protocol to the patients.

\section{Inclusion and Exclusion Criteria}

The sample constituted of the general population and health care workers who suffered from COVID-19 disease at least 3 months ago. Exclusion criteria implied participants of the pilot study and those unwilling to participate. The prevalidated reliable questionnaire adapted from relevant studies and literature available was used. It was piloted on a population of medical practitioners.

\section{Measures and Variables}

The final Questionnaire comprised of three sections, both paper-based and web-based survey (English and Urdu) with preferably nonmedical terms for greater compliance. Feedback was obtained from $(62 \%)$ participants. Section - I focused on the demographic profile (Age, Gender, Profession) of the participants. Section - II was designed to investigate COVID-19 disease history, comorbid, coronavirus disease severity along with the number of individuals infected in the family, duration of positive polymerase chain reaction results, and management destination (Hospital or Home). Section - III addressed the frequency of persistent symptoms (Gastrointestinal, Systemic, Cardiovascular, Musculoskeletal, Immunological, Dermatological, Neuropsychiatric, Ophthalmological, Pulmonary, Otorhinolaryngological, Endocrine, Reproductive and Genitourinary systems).

\section{E. Analysis}

Data was entered and analyzed by using data management software IBM SPSS (version 23.0). The descriptive statistics of a continuous variable (i.e., age) were presented as mean and standard deviation, while for categorical data (i.e., vaccination status, comorbidities, post-vaccination side effects) frequencies and percentages were used. Postvaccination side effects were compared among categorical groups including gender, comorbidities, and history of COVID-19 infection by using the Chi-Square test, whereas post-vaccination side effects were compared with age using independent samples T-test. A p-value of $\leq 0.05$ was statistically significant.

\section{RESULTS}

TABLE I: DEMOGRAPHIC AND CLINICAL CHARACTERISTICS OF STUDY RESPONDERS ( $\mathrm{N}=168)$

\begin{tabular}{|c|c|c|c|}
\hline \multicolumn{2}{|c|}{ Characteristics } & $\begin{array}{c}\text { Frequency } \\
\text { (n) }\end{array}$ & $\begin{array}{c}\text { Percentage } \\
(\%)\end{array}$ \\
\hline \multirow{5}{*}{ Age group in years } & $15-25$ & 20 & $11.9 \%$ \\
\hline & $26-35$ & 52 & $31.0 \%$ \\
\hline & $36-45$ & 60 & $35.7 \%$ \\
\hline & $46-55$ & 20 & $11.9 \%$ \\
\hline & $>55$ & 16 & $9.5 \%$ \\
\hline \multirow{2}{*}{ Gender } & Male & 48 & $28.6 \%$ \\
\hline & Female & 120 & $71.4 \%$ \\
\hline \multirow{3}{*}{ Status } & Working & 96 & $57.1 \%$ \\
\hline & Unemployed & 52 & $31.0 \%$ \\
\hline & Students & 20 & $11.9 \%$ \\
\hline \multirow{5}{*}{ Profession } & Students & 20 & $11.9 \%$ \\
\hline & Teachers & 16 & $9.5 \%$ \\
\hline & $\begin{array}{l}\text { Government } \\
\text { employees }\end{array}$ & 44 & $26.2 \%$ \\
\hline & $\begin{array}{c}\text { Medical } \\
\text { professionals }\end{array}$ & 36 & $21.4 \%$ \\
\hline & Housewives & 52 & $31.0 \%$ \\
\hline \multirow{2}{*}{$\begin{array}{l}\text { Prolonged COVID } \\
\text { Disease Symptoms }\end{array}$} & No & 36 & $21.4 \%$ \\
\hline & Yes & 132 & $78.6 \%$ \\
\hline \multirow{4}{*}{ Comorbidities } & None & 132 & $78.6 \%$ \\
\hline & Asthma & 8 & $4.8 \%$ \\
\hline & Hypertension & 20 & $11.9 \%$ \\
\hline & Diabetes & 8 & $4.8 \%$ \\
\hline \multirow{4}{*}{ Disease severity } & Mild & 84 & $50.0 \%$ \\
\hline & $\begin{array}{l}\text { Mild- } \\
\text { moderate }\end{array}$ & 48 & $28.6 \%$ \\
\hline & $\begin{array}{l}\text { Moderate- } \\
\text { severe }\end{array}$ & 32 & $19.0 \%$ \\
\hline & Severe & 4 & $2.4 \%$ \\
\hline \multirow[b]{2}{*}{ Management } & Home-based & 140 & $83.3 \%$ \\
\hline & $\begin{array}{c}\text { Hospital- } \\
\text { based }\end{array}$ & 28 & $16.7 \%$ \\
\hline \multirow{3}{*}{$\begin{array}{c}\text { Family members } \\
\text { infected }\end{array}$} & None & 36 & $21.4 \%$ \\
\hline & $1-3$ & 80 & $47.6 \%$ \\
\hline & $>3$ & 52 & $31.0 \%$ \\
\hline \multirow{5}{*}{$\begin{array}{l}\text { Negative PCR for } \\
\text { COVID-19 infection }\end{array}$} & 7 days & 40 & $23.8 \%$ \\
\hline & 10 days & 32 & $19.0 \%$ \\
\hline & 14 days & 56 & $33.3 \%$ \\
\hline & 21 days & 24 & $14.3 \%$ \\
\hline & $>21$ days & 16 & $9.5 \%$ \\
\hline
\end{tabular}

Table I, 168 responders participated in this survey study. Overall, there were $48(28.6 \%)$ males while $120(71.4 \%)$ females. The majority of the responders, $60 \quad(35.7 \%)$ belonged to the age group of 36-45 years followed by 53 (31.0\%) of 26-35-year age. There were $20(11.9 \%)$ students among the study participants, $96(57.1 \%)$ workers while the remaining $52(31.0 \%)$ were unemployed. About 36 (11.9\%) 
were found to be suffering from comorbidity (asthma, hypertension, and diabetes). It was reported that half of the responders, $84(50.0 \%)$ suffered from mild disease symptoms, while only $4(2.4 \%)$ experienced the severe disease. The disease was managed at home for a major chunk of responders, 140 (83.3\%). About 36 (21.4\%) responders reported that their family members were not infected by the virus while they suffered from COVID-19 infection. The summary of demographic and clinical characteristics is given in Table I.

Among 168 participants who suffered from COVID-19 infection, around $132(78.6 \%)$ were found to have suffered from long COVID syndrome with the persistence of symptoms beyond four weeks of infection. For about 56 (33.3\%) participants, PCR test for COVID-19 infection was detected negative within 14 days of infection, while for 16 (9.5\%) participants it took more than 21 days to obtain a negative PCR result as shown in Fig. 1.

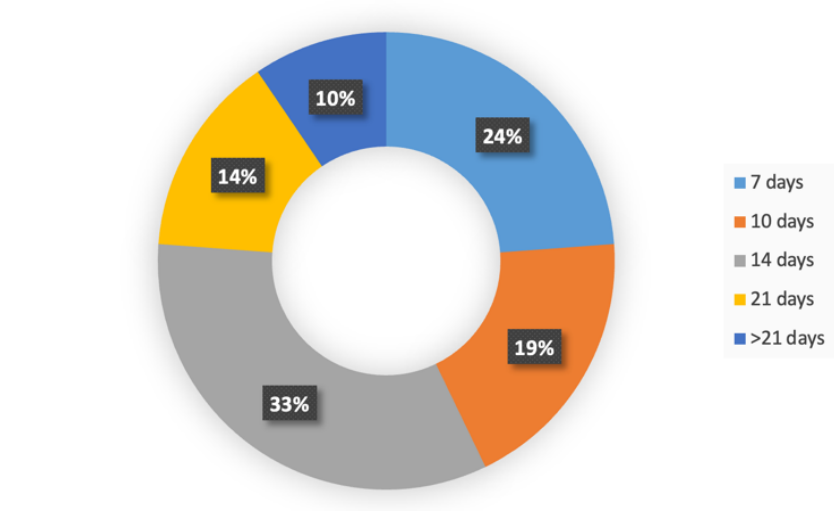

Fig. 1. Time to detect a negative PCR for COVID-19 infection among study participants.

The frequency and percentage of symptoms of long COVID syndrome, classified according to the involvement of the system, are shown in Fig. 2 in detail. The most common symptoms included fatigue $(78.8 \%)$, fever $(60.0 \%)$, loss of taste $(57.8 \%)$, dry cough $(45.5 \%)$, headache (42.4\%), post-exertional malaise $(45.5 \%)$, shortness of breath $(39.4 \%)$, insomnia $(39.0 \%)$, loss of smell $(36.4 \%)$, loss of appetite (33.3\%) and depression (33.3\%).

Table II shows the relationship of demographic/clinical characteristics of participants with risk of experiencing long COVID syndrome with symptoms persisting for more than 4 weeks. The male gender was more likely to have experienced long COVID syndrome as compared to females $(p=0.009)$, similarly, the higher age group was more likely to have suffered from long term COVID-19 symptoms $(p<0.001)$. Participants with existing chronic illnesses were also more likely to have experienced persistent COVID-19 symptoms beyond four weeks $(\mathrm{p}<0.001)$. At the same time, moderate and severe disease severity was also found to be linked with long-term COVID-19 symptoms as compared to mild disease $(p<0.001)$. Those who were isolated in hospitals were more likely to experience long COVID syndrome as compared to those isolated at home $(\mathrm{p}=0.002)$. It was found that participants who had a longer period of infection, which means for whom negative PCR was detected at 21 days or $>21$ days, were more likely to have suffered from long COVID syndrome $(\mathrm{p}<0.001)$. There were 40 participants for whom PCR took 21 or more days to give negative results, and $90 \%$ of such patients developed long COVID syndrome as shown in Table II.

Depression $(p<0.001)$ and insomnia $(p<0.001)$ was more commonly found in patients isolated at hospitals as compared to home, depression $(p<0.001)$ and insomnia $(p=0.044)$ was more common among females as compared to males and participants belonging to age group 26-45 years $(\mathrm{p}=0.017, \mathrm{p}=0.008)$.

TABLE II: RELATIONSHIP OF LONG COVID SYNDROME WITH DEMOGRAPHIC/CLINICAL CHARACTERISTICS

\begin{tabular}{|c|c|c|c|c|}
\hline \multirow{2}{*}{\multicolumn{2}{|c|}{ Characteristics }} & \multicolumn{2}{|c|}{$\begin{array}{c}\text { Symptoms persisted beyond } \\
4 \text { weeks }\end{array}$} & \multirow[t]{2}{*}{$\mathrm{p}$} \\
\hline & & Yes & No & \\
\hline \multirow{5}{*}{$\begin{array}{l}\text { Age group } \\
\text { in years }\end{array}$} & $15-25(n=20)$ & $8(40.0 \%)$ & $12(60.0 \%)$ & \multirow{5}{*}{$<0.001$} \\
\hline & $26-35(n=52)$ & $52(100.0 \%)$ & $0(0.0 \%)$ & \\
\hline & $36-45(n=60)$ & $44(73.3 \%)$ & $16(26.7 \%)$ & \\
\hline & $46-55(n=20)$ & $16(80.0 \%)$ & $4(20.0 \%)$ & \\
\hline & $>55(n=16)$ & $12(75.0 \%)$ & $4(25.0 \%)$ & \\
\hline \multirow[b]{2}{*}{ Gender } & Male $(n=48)$ & $44(91.7 \% \%)$ & $4(8.3 \%)$ & \multirow[b]{2}{*}{0.009} \\
\hline & $\begin{array}{l}\text { Female } \\
(n=120)\end{array}$ & $88(73.3 \%)$ & $32(26.7 \%)$ & \\
\hline \multirow{2}{*}{ Comorbid } & Yes $(n=36)$ & $36(100.0 \%)$ & $0(0.0 \%)$ & \multirow{2}{*}{$<0.001$} \\
\hline & No $(n=132)$ & $96(72.7 \%)$ & $36(27.3 \%)$ & \\
\hline \multirow{4}{*}{$\begin{array}{l}\text { Disease } \\
\text { severity }\end{array}$} & Mild $(n=84)$ & $52(61.9 \%)$ & $32(38.1 \%)$ & \multirow{4}{*}{$<0.00$} \\
\hline & $\begin{array}{l}\text { Mild-moderate } \\
\qquad(\mathrm{n}=48)\end{array}$ & $44(91.7 \%)$ & $4(8.3 \%)$ & \\
\hline & $\begin{array}{c}\text { Moderate- } \\
\text { severe }(n=32)\end{array}$ & $32(100.0 \%)$ & $0(0.0 \%)$ & \\
\hline & Severe $(n=4)$ & $4(100.0 \%)$ & $0(0.0 \%)$ & \\
\hline \multirow{2}{*}{ Management } & $\begin{array}{l}\text { Home-based } \\
(n=140)\end{array}$ & $104(74.3 \%)$ & $36(25.7 \%)$ & \multirow{2}{*}{0.002} \\
\hline & $\begin{array}{l}\text { Hospital-based } \\
(\mathrm{n}=28)\end{array}$ & $28(100.0 \%)$ & $0(0.0 \%)$ & \\
\hline \multirow{5}{*}{ PCR } & 7 days $(n=40)$ & $24(60.0 \% \%)$ & $16(40.0 \%)$ & \multirow{5}{*}{$<0.001$} \\
\hline & 10 days $(\mathrm{n}=32)$ & $20(62.5 \%)$ & $12(37.5 \%)$ & \\
\hline & 14 days $(\mathrm{n}=56)$ & $52(92.9 \%)$ & $4(7.1 \%)$ & \\
\hline & 21 days $(n=24)$ & $24(100 \%)$ & $0(0.0 \%)$ & \\
\hline & $\begin{array}{c}>21 \text { days } \\
(\mathrm{n}=16)\end{array}$ & $12(75.0 \%)$ & $4(25.0 \%)$ & \\
\hline
\end{tabular}

\section{DisCUSSION}

The lasting effects of COVID-19 disease are coming out as a new dilemma for patients and healthcare workers alike. This prolonging of disease has been named "Long-COVID" or "Long-Haulers". In the field of medicine, commencing a disease is termed acute while an illness occurring for 3 weeks is entitled chronic [12]. Multiorgan symptoms after COVID-19 such as cough and short breathing, fatigue, headache, palpitations, chest pain, joint pain, physical limitations, depression, and insomnia, are increasingly being stated by the affecters of varying ages [13].

At the Lancet-Chinese Academy of Medical Sciences conference held on Nov 23, Bin Cao presented data on longterm outcomes of COVID-19 for patients in Wuhan and warned that dysfunctions and problems could persist in some discharged patients at least for 6 months. So-called long COVID is a growing health concern hence due to complexities and variabilities of disease course and accepting the potential scale, concrete measures are needed to address as it could evade years of struggle for management of patients with long COVID [14], [15]. 


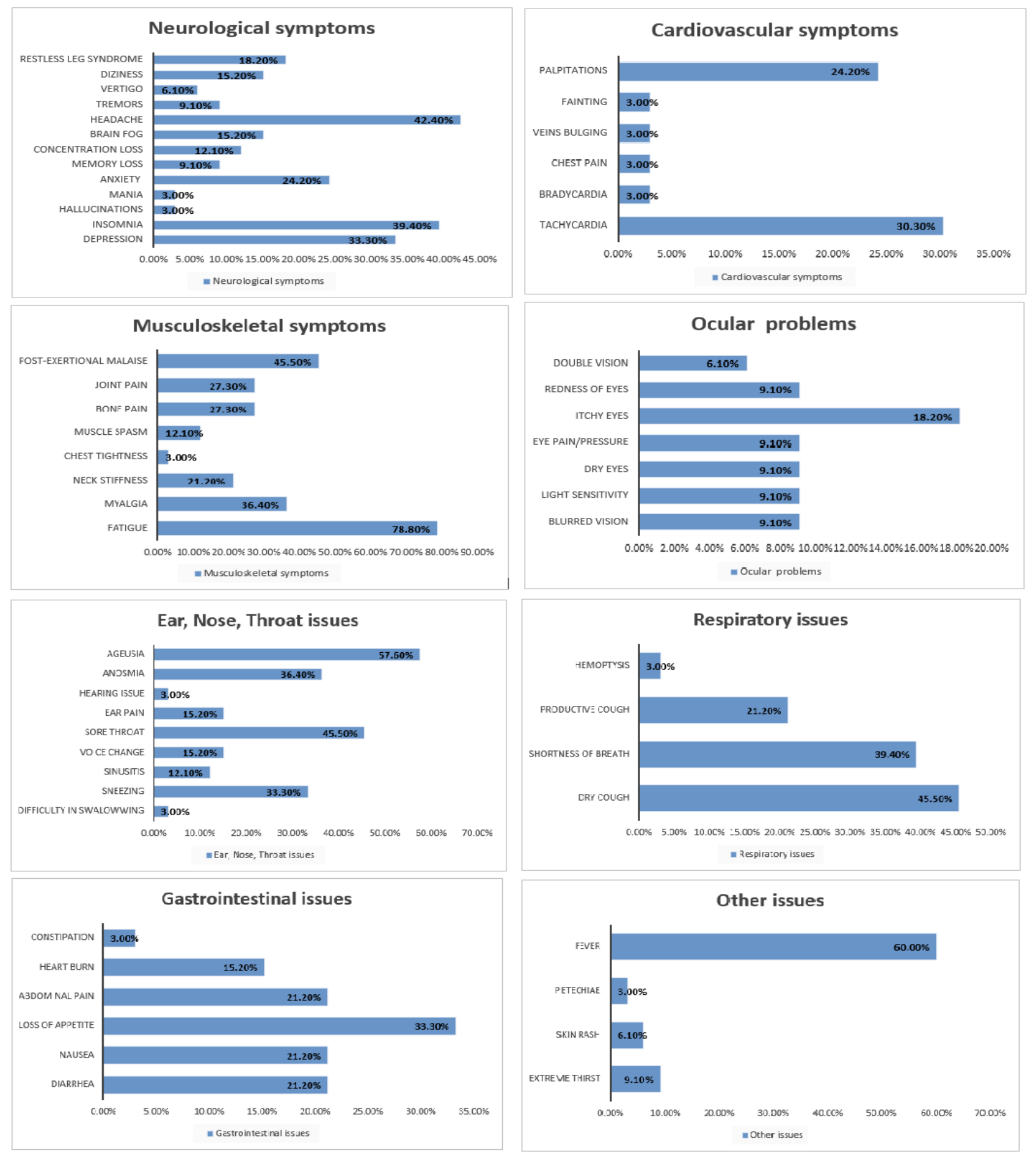

Fig. 2. Frequency of symptoms that persisted more than four weeks after COVID-19 infection ( $\mathrm{n}=132)$.

Reference [16] reported that females were considerably more likely to report symptoms of myalgia and fatigue, when matched to males $(p=<0.001)$. No significant changes between genders were observed for low mood, anxiety, and sleep disturbance, and memory impairment, attention deficit, and cognitive impairment [16]. When compared with this study results depression $(\mathrm{p}<0.001)$ and insomnia $(\mathrm{p}=0.044)$ were more common among females as compared to males.

Long COVID (symptoms past 3 weeks) and Long-Lasting Post-COVID disease (beyond 12 weeks) are multisystem syndromes and need a multidimensional approach to tackle the physical, cognitive, psychological, social, and vocational domains of this health situation [17], [18].
Lungs and heart could damage in long COVID-19, but the degree and length of this damage need to be further validated. Therefore, need for a long-term check-up of COVID-19 patients tied with rehabilitation therapy services to those with persistent or new symptoms. Although vaccination has become the instant focus of pandemic response for many countries, patients with long COVID must not be overlooked or sidelined as countries begin to consider the end of the pandemic [19].

These patients would in need of multidisciplinary care and general physicians will be focal practitioners in cognitive rehabilitation programs, emotional support groups and prolonged follow ups are significant to help this suffering patients to get back to their normal lives. General 
Practice is back bone of chronic diseases management therefore when major burden of the disease will be over due to mass vaccination programs there will be a shift of burden from intensive care to primary care to restore the tormented patients with prolonged issues of Long COVID syndrome and disease. There is a need for more investigation in this area, especially in low-and-middle-income countries for making strategies and achieve preparedness to combat this new health pandemic and disaster. Unfortunately, psychiatric, and chronic health conditions management and facilities are already debilitated and tempered in Pakistan with weak infrastructure of general practice, hence this dilemma can be painstakingly difficult to handle.

\section{LimitATIONS AND STRENGTHS}

To establish definitive statistics more studies on larger sample sizes are to be carried out. Due to the uncooperative attitude of the probable participants, limited individuals gave consent and feedback. COVID - 19 is undergoing rapid mutations hence Long COVID Syndrome could be more pronounced with a particular strain and risk stratification to be established but due to constrained laboratory facilities randomization couldn't be carried out according to strain. This study unravels a crucial phenomenon associated with this COVID disease with some insight about the pattern of syndrome.

\section{CONCLUSION}

In conclusion, this study has shown that persistence of COVID disease symptoms (most common symptoms included fatigue, fever, loss of taste, dry cough, headache, post-exertional malaise, shortness of breath, insomnia, loss of smell, loss of appetite, and depression) are common in Pakistani population. Male gender, existing chronic illnesses, moderate and severe disease, higher age group, and isolation at the hospital are the probable risk factors.

\section{REFERENCES}

[1] Taribagil P, Creer D, Tahir H. 'Long COVID' syndrome. BMJ Case Rep. 2021;14(4): e241485.

[2] Aiyegbusi OL, Hughes SE, Turner G, Rivera SC, McMullan C, Chandan JS, et al. Symptoms, complications and management of long COVID: a review. J. R. Soc. Med. 2021; 0(0): 1-15.

[3] Menges D, Ballouz T, Anagnostopoulos A, Aschmann HE, Domenghino A, Fehr JS, et al. Burden of post-COVID-19 syndrome and implications for healthcare service planning: a population-based cohort study. PLoS One. 2021; 16(7): e0254523.

[4] Tenforde MW, Kim SS, Lindsell CJ, Billig Rose E, Shapiro NI, Files $\mathrm{DC}$, et al. Symptom duration and risk factors for delayed return to usual health among outpatients with COVID-19 in a multistate health care systems network — United States, 2020. MMWR. 2020; 69(30): 993-8.

[5] Carvalho SC, Laurent E, Lemaignen A, Beaufils E, Bourbao-Tournois C, Laribi S, et al. Follow-up of adults with noncritical COVID-19 two months after symptom onset. Clin Microbiol Infect. 2021; 27(2): 258263.

[6] Callard F, Perego E. How and why patients made long Covid. Soc Sci Med 2021; 268: 113426.

[7] Yelin D, Margalit I, Yahav D, Runold M, Bruchfeld J. Long COVID19 it's not over until. Clin Microbiol Infect. 2021; 27(4): 506-508.

[8] Becker RC. COVID-19 and its sequelae: a platform for optimal patient care, discovery, and training. J Thromb Thrombolysis. 2021; 51(3): 587-594.
[9] Halpin S, O'Connor R, Sivan M. Long COVID, and chronic COVID syndromes. J Med Virol. 2021; 93(3): 1242-1243.

[10] Garg M, Maralakunte M, Garg S, Dhooria S, Sehgal I, Bhalla AS et al. The conundrum of 'Long-COVID-19': a narrative review. Int $J$ Gen Med. 2021; 14: 2491-2506.

[11] Davis HE, Assaf GS, McCorkell L, Wei H, Low RJ, Re'em Y. et al. Characterizing long COVID in an international cohort: 7 months of symptoms and their impact. E Clinical Medicine. 2020: 101019.

[12] Baig AM. Deleterious outcomes in long-hauler COVID-19: the effects of SARS-CoV-2 on the CNS in chronic COVID syndrome. ACS Chem Neurosci. 2020; 11(24): 4017-4020.

[13] Mahase E. Long covid could be four different syndromes, the review suggests. BMJ. 2020; 371: m3981.

[14] The Lancet. Facing up to long COVID. Lancet. 2020; 396(10266): 1861.

[15] Nabavi N. Long covid: How to define it and how to manage it. BMJ. 2020; 370: m3489.

[16] Sykes DL, Holdsworth L, Jawad N, Gunasekera P, Morice AH, Crooks MG. Post-COVID-19 symptom burden: what is long-covid and how should we manage it? Lung. 2021; 199(2): 113-119.

[17] Halpin S, O'Connor R, Sivan M. Long COVID, and chronic COVID syndromes. J Med Virol. 2021; 93(3): 1242-1243.

[18] Butler MJ, Barrientos RM. The impact of nutrition on COVID-19 susceptibility and long-term consequences. Brain Behav Immun. 2020; 87: 53-54

[19] Iwu CJ, Iwu CD, Wiysonge CS. The occurrence of long COVID: a rapid review. Pan Afr Med J. 2021; 38:65. 